\title{
THE REGULAR RADICAL OF SEMIGROUP RINGS OF COMMUTATIVE SEMIGROUPS
}

\author{
by A. V. KELAREV
}

(Received 21 August, 1990)

A description of regular group rings is well known (see [12]). Various authors have considered regular semigroup rings (see [17], [8], [10], [11], [4]). These rings have been characterized for many important classes of semigroups, although the general problem turns out to be rather difficult and still has not got a complete solution. It seems natural to describe the regular radical in semigroup rings for semigroups of the classes mentioned. In [10], the regular semigroup rings of commutative semigroups were described. The aim of the present paper is to characterize the regular radical $\rho(R[S])$ for each associative ring $R$ and commutative semigroup $S$.

We shall apply the approach to the investigations of these rings elaborated by a number of authors ([3], a survey). It is based on the decomposition of a commutative semigroup into the union of its Archimedean subsemigroups. Recall that a commutative semigroup $S$ is Archimedean if and only if, for any $s, t \in S$, there is a natural number $n$ such that $s^{n} \in S^{1} t$. In Section 1, the radical $\rho(R[S])$ is described for an Archimedean $S$; Section 2 is devoted to the general case.

So far that approach has been used only for some super-nilpotent radicals, i.e. radicals whose classes contain all the nilpotent rings. The regular radical is not super-nilpotent. This brings about esssential distinctions between its behaviour and that of the radicals investigated earlier. For example, it is impossible to reduce the description of $\rho(R[S])$ to the case where $S$ is separative, as it has been done for all the other radicals (see [3]). Another few differences will be pointed out in Section 2.

1. Archimedean semigroups. First of all we record two lemmas which will illuminate the main result of this section. All semigroups considered are commutative.

Lemma 1 (see [1, §4.3, Exercise 5]). If $S$ a periodic Archimedean semigroup then it contains a unique idempotent $e$ and the ideal $e S$ is the largest subgroup of $S$.

LeMma 2. If $R$ is a regular ring and $\pi$ is a set of primes then there exists a largest ideal $I$ in $R$ such that the additive period of any element of I has no divisor in $\pi$.

THEOREM 1. Let $S$ be an Archimedean commutative semigroup, $R$ an arbitrary ring. If $S$ is not periodic then the regular radical $\rho(R[S])$ is equal to zero. If $S$ is periodic then $\rho(R[S])=I[H]$, where $H$ is the largest subgroup of $S$ and $I$ is the largest ideal of $\rho(R)$ such that the additive period of any element of $I$ is not divisible by any prime that is the order of an element in $\mathrm{H}$.

Proof of Lemma 1. Let $S$ be a periodic Archimedean semigroup. Each periodic semigroup contains an idempotent. Let $e$ denote an idempotent of $S$. Since $S$ is Archimedean, for any idempotent $f$ in $S$, we have $f=f^{n} \in e S$ and $e \in f S$, whence $f=f e=e$. So $e$ is the only idempotent in $S$. Given that $S$ is periodic, for any $x \in S$, there exists $n$ such that $x^{n}=e$. Hence, for $x \in e S$, the subsemigroup generated by $x$ is a group. Thus $e S$ is a group. Evidently, it contains every subgroup $G$ of $S$, because $e$ must be the identity of $G$ and $G=e G$. 
Proof of Lemma 2. Let $M$ designate the set of all ideals in $R$ such that there is not any prime in $\pi$ which divides the additive period of an element of these ideals. Obviously $M$ is not empty, since it contains the zero ideal of $R$. Setting $I=\sum_{j \in M} J$, we claim that $I \in M$. Each element of $I$ belongs to a finite sum of ideals from $M$. Therefore we have to prove that every finite sum of this sort is in $M$. Obviously, it suffices to consider a sum of two ideals. Let $A, B \in M$. Take any $c \in A+B$, say $c=a+b, a \in A, b \in B$. Suppose that $p c=0$ for some $p \in \pi$. By the regularity of $R$, there exists $d \in R$ such that ada=a. Putting $e=a d$, we get $e c \in A$ and $p e c=0$. Since $A \in M$, it follows that $e c=0$ implying $a=e a=-e b \in B$. Therefore $c \in B$, a contradiction since $B \in M$. Thus $I \in M$, that is $I$ is the largest ideal in $M$.

Remark. The analogue of Lemma 2 for non-regular rings is not valid.

For the proof of Theorem 1 several known results are needed.

Leмma 3 [10]. Let $R$ be an algebra over a field of characteristic $p$ (a prime or 0 ), and let $S$ be a commutative semigroup. Then $R[S]$ is regular if and only if $R$ is regular and $S$ is a union of finite groups whose orders are not divisible by $p$.

The set of all elements $\sum_{i=1}^{n} r_{i} s_{i} \in R[S]$ such that $\sum_{i=1}^{n} r_{i}=0$ is called the augmentation ideal of $R[S]$ and will be denoted by $\operatorname{Aug}(R[S])$. The following lemma is well known, see for instance [12].

Lemma 4. If $G$ is a p-group and $p R=0$ then $\operatorname{Aug}(R[G])$ is a nil ideal.

A commutative semigroup $S$ is said to be separative if $s, t \in S, s^{2}=s t=t^{2}$ imply $s=t$. There exists a least congruence on $S$ whose factor semigroup is separative; denote it by $\xi$. Let $I(R, S, \xi)$ represent the ideal of $R[S]$ consisting of all sums $\sum_{i=1}^{n}\left(r_{i} s_{i}-r_{i} t_{i}\right)$, where $r_{i} \in R,\left(s_{i}, t_{i}\right) \in \xi$.

LEMMA 5 [9]. $I(R, S, \xi)$ is a nil ideal.

The following lemma is analogous to [12, Lemma 7.1.3].

LEMMA 6. If $H$ is a subgroup of a group $G$ then $\rho(R[H]) \supseteq R[H] \cap \rho(R[G])$.

Proof. Take any $x \in R[H] \cap \rho(R[G])$. Then $x y x=x$ for some $y \in R[G]$, say $y=$ $\sum_{g \in G} y_{g} g$. Since $R[G]$ is a direct sum of $R$-modules $R[H]$ and $R[G \backslash H]$, and $x y_{g} g x \in R[G \backslash H]$ for any $g \in G \backslash H$, then $\sum_{g \in G \backslash H} x y_{g} g x=0$. Hence $x z x=x$, where $z=\sum_{g \in H} y_{g} g \in R[H]$. Thus $R[H] \cap \rho(R[G])$ is a regular ideal of $R[H]$.

LEMMA 7. If $G$ is the infinite cyclic group then $\rho(R[G])=0$.

Proof. Let $g$ denote a generator of $G$. Assume $\rho(R[G]) \neq 0$ and choose nonzero $x=\sum_{i=-\infty}^{+\infty} x_{i} g^{i} \in \rho(R[G])$ such that the number of nonzero summands $x_{i} g^{i}$ is minimal. Let $x=\sum_{i=m}^{n} x_{i} g^{i}$, where $m \leqslant n, x_{m} \neq 0, x_{n} \neq 0$. 
First we consider the case when $m=n$. Setting $r=x_{m}, y=r g+r g^{2}$, we get $y=x g^{1-m}+x g^{2-m} \in \rho(R[G])$, because $\rho(R[G])$ is an ideal of $R^{1}[G]$. Hence there exists $z=\sum_{i=1}^{k} r_{i} h_{i} \in R[G]$ such that $y z y=y, r_{i} \in R, r_{i} \neq 0, h_{i} \in G$, and $h_{1}, \ldots, h_{k}$ are pairwise distinct. If $r r_{i} r=0$ for some $i$ then $y r_{i} h_{i} y=0$ and we may take away the summand $r_{i} h_{i}$ from $z$. Therefore we may assume that $r r_{i} r \neq 0$ for each $i$. Let $a$ and $b$ be, respectively, the least and greatest numbers such that $g^{a}, g^{b} \in\left\{h_{1}, \ldots, h_{k}\right\}$, and let $g^{a}=h_{c}, g^{b}=h_{d}$. Then $r r_{c} r g^{a+2}$ and $r r_{d} r g^{b+4}$ occur in $y z y$. Therefore $1 \leqslant a+2$ and $b+4 \leqslant 2$, which contradicts $a \leqslant b$.

Now suppose that $m<n$. There exists $y=\sum_{i=1}^{k} r_{i} h_{i} \in R[G]$ such that $x y x=x, r_{i} \in R$, $r_{i} \neq 0, h_{i} \in G$ and $h_{1}, \ldots, h_{k}$ are pairwise distinct. We may assume that $x r_{i} h_{i} x \neq 0$ for each $i$ because otherwise it would be possible to throw away the summand $r_{i} h_{i}$ of $y$. Now fix some $i, 1 \leqslant i \leqslant k$. Then $x r_{i} h_{i} x_{j} g^{j} \neq 0$ for some $j$. If $x_{n} g^{n} r_{i} h_{i} x_{j} g^{j}=0$ then $x r_{i} h_{i} x_{j} g^{j}$ has a smaller number of summands than $x$, which is a contradiction since $x r_{i} h_{i} x_{j} g^{j} \in \rho(R[G])$. So $x_{n} g^{n} r_{i} h_{i} x_{j} g^{j} \neq 0$, and hence $x_{n} g^{n} r_{i} h_{i} x \neq 0$, from which it follows by a similar argument that $x_{n} g^{n} r_{i} h_{i} x_{n} g^{n} \neq 0$. Likewise one can show that $x_{m} g^{m} r_{i} h_{i} x_{m} g^{m} \neq 0$. Let $M$ be the set of integers $l$ such that $g^{l}=h_{i}$ for some $i \in\{1, \ldots, k\}$. Denote by $a$ and $b$ the least and greatest numbers in $M$, respectively. Let $g^{a}=h_{c}, g^{b}=h_{d}$. Then the summands $\left(x_{m} r_{c} x_{m}\right) g^{2 m+a}$ and $\left(x_{n} r_{d} x_{n}\right) g^{2 n+b}$ occur in $x y x$. Hence $m \leqslant 2 m+a, 2 n+b \leqslant n$ in contradiction to $m<n$. Thus the equality $x y x=x$ is impossible.

Lemma 8. Let $S$ be a cancellative semigroup, $G$ the group of quotients of $S$. Then $\rho(R[G]) \supseteq \rho(R[S])$.

Proof. Let $I$ be the ideal generated in $R[G]$ by $\rho(R[S])$. We claim that $I$ is regular.

Take any $x \in I$. There exist $r_{1}, \ldots, r_{m} \in \rho(R[S]), a_{1}, \ldots, a_{m}, b_{1}, \ldots, b_{m} \in R[G]$, $a_{j}^{(i)}, b_{j}^{(i)} \in R[G], i=1, \ldots, m, j=1, \ldots, m_{i}$, and integers $n_{1}, \ldots, n_{m}$ such that

$$
x=\sum_{i=1}^{m}\left(n_{i} r_{i}+a_{i} r_{i}+r_{i} b_{i}+\sum_{j=1}^{m_{i}} a_{j}^{(i)} r_{i} b_{j}^{(i)}\right) .
$$

For each $g \in G$, fix elements $s, t$ in $S$ such that $g=s t^{-1}$. This $t$ will be called the denominator of $g$. Let $w$ denote the product of all denominators of elements of $G$ occurring in the supports of $a_{i}, b_{i}, a_{j}^{(i)}, b_{j}^{(i)}$. Then $a_{i} w^{2}, b_{i} w^{2}, a_{j}^{(i)} w, b_{j}^{(i)} w \in R[S]$. Besides, $n_{i} r_{i} w^{2} \in \rho(R[S])$ since the radical is an ideal in $R^{1}[S]$. Hence $x w^{2} \in \rho(R[S])$, and so $x w^{2} y x w^{2}=x w^{2}$ for some $y \in R[S]$. Setting $z=y w^{2}$, we get $z \in R[G]$ and $x z x=$ $x w^{2} y x w^{2} w^{-2}=x w^{2} w^{-2}=x$. We have shown that $I$ is regular. Therefore $\rho(R[G] \supseteq I \supseteq$ $\rho(R[S])$ and the proof is complete.

LeMma 9. If $S$ is a non-periodic Archimedean semigroup then $\rho(R[S])=0$.

Proof. Suppose to the contrary that $\rho(R[S]) \neq 0$. Lemma 5 ensures that $I(R, S, \xi) \cap \rho(R[S])=0$. Hence

$$
\rho(R[S / \xi]) \cong \rho(R[S] / I(R, S, \xi)) \supseteq[\rho(R[S])+I(R, S, \xi)] / I(R, S, \xi) \neq 0
$$

and we may assume that from the very beginning $S=S / \xi$, i.e. $S$ is separative. By [1, Theorem 4.16], every separative Archimedean semigroup is cancellative. Lemma 8 
implies $\rho(R[G]) \neq 0$, where $G$ is the group of quotients of $S$. Take any nonzero $x \in \rho(R[G])$, say $x=\sum_{i=1}^{n} r_{i} g_{i}, r_{i} \in R, g_{i} \in G$. Let $H$ denote the group generated in $G$ by $g_{1}, \ldots, g_{n}$ and a non-periodic element of $S$. By Lemma $6, x \in \rho(R[H])$. Each finitely generated Abelian group is known to be a direct product of finitely many cyclic groups. Since $H$ is infinite, there is a group $D$ and an infinite cyclic group $C$ such that $H \cong C \times D$. Therefore $R[H] \cong(R[D])[C]$ and Lemma 7 implies $\rho(R[H])=0$, a contradiction.

Leмma 10. If a prime $p$ divides the order of a finite Abelian group $G$ and $p R=0$ then $\rho(R[G])=0$.

Proof. Let $H$ be the largest $p$-subgroup of $G$. Then $G=H \times N$ for a group $N$. Put $A=R[N]$. Clearly $R[G] \cong A[H]$. We have to prove that $\rho(A[H])=0$.

Denote the elements of $H$ by $h_{1}, \ldots, h_{n}$. Take any nonzero $x$ in $\rho(A[H])$, say $x=\sum_{i=1}^{n} a_{i} h_{i}$, where $a_{i} \in A$. By Lemma $4, \operatorname{Aug}(A[H]) \cap \rho(A[H])=0$ and so $\sum_{i=1}^{n} a_{i} \neq 0$. Hence, setting $w=h_{1}+\ldots+h_{n}, y=x w$, we obtain $y=w \sum_{i=1}^{n} a_{i} \neq 0$ and $y \in \rho(A[H])$. However, $y \in \operatorname{Aug}(A[H])$ since $p$ divides $n$, giving a contradiction. Thus $\rho(A[H])=0$.

Lemma 11. If $S$ is an Archimedean semigroup and $\rho(R)=0$ then $\rho(R[S])=0$.

Proof. As at the beginning of the proof of Lemma 9, it suffices to consider the case where $S$ is cancellative. By Lemma 9, we may assume that $S$ is periodic. It is known that every cancellative periodic Archimedean semigroup $S$ is a group. (Indeed, $S$ contains an idempotent $e ; e s=e(e s)$ implies $s=e s$ for each $s$, and so $e$ is the identity of $S$; every $s$ in $S$ is invertible since $e^{n} \in s S^{1}$ for some $n$.) Thus $S$ is a group.

Suppose that there exists a nonzero element $x$ in $\rho(R[S])$. Let $H$ be the subgroup generated in $S$ by all elements in the support of $x$. Since $S$ is periodic, $H$ is finite. By Lemma $6, x \in \rho(R[H]) \neq 0$. Denote by $P$ the set of additively periodic elements of $R$. Clearly $P$ is an ideal of $R$. The following two cases are possible.

Case 1. There exists a nonzero $y$ in $P[H] \cap \rho(R[H])$. Let $n$ be the least natural number such that $n y=0$. Take a prime divisor $p$ of $n$ and set $m=n / p, z=m y$, $D=\{r \in R \mid p r=0\}$. Then $z \in \rho(R[H]) \cap D[H], z \neq 0$. Since $D[H]$ is an ideal of $R[H]$ and $\rho$ is hereditary, $z \in \rho(D[H])$. Evidently $D[H]$ is an algebra over the prime field of characteristic $p$. If $p$ divides $|H|$ then $\rho(D[H])=0$ by Lemma 10. If $p$ does not divide $|H|$ then $|H|$ is invertible in $D$, and [11, Theorems 1 and 2], yields $\rho(D[H])=\rho(D)[H]=0$, a contradiction.

Case 2. $P[H] \cap \rho(R[H])=0$. Passing to the quotient ring $R[H] / P[H] \cong(R / P)[H]$, we may assume that from the very beginning $P=0$ and $\rho(R[H]) \neq 0$. Further, $\rho\left(R^{1}[H]\right) \supseteq \rho(R[H]) \neq 0$. However, [11, Theorems 1 and 2] show that $\rho\left(R^{1}[H]\right)=$ $\rho\left(R^{1}\right)[H]=0$.

Thus in both the cases we have got a contradiction. Therefore $\rho(R[S])=0$.

LEMMA 12. Each regular ring whose additive group is periodic is a direct sum of algebras over fields. 
Proof. Let $R$ be regular. Denote by $\pi$ the set of all primes. For $p \in \pi$, let $R_{p}$ denote the set of elements $x \in R$ such that $p^{k} x=0$ for some $k$. We claim that $R_{p}$ is an algebra over the field with $p$ elements. To this end it suffices to check that $p R_{p}=0$. Suppose $x \in R_{p}, x \neq 0$. Let $k$ be the least natural number such that $p^{k} x=0$. Then $p^{k-1} x \neq 0$ and $p^{k-1} x=\left(p^{k-1} x\right) y\left(p^{k-1} x\right)$ for some $y \in R$. If $k>1$ then $2 k-2 \geqslant k$ and so $p^{k-1} x y p^{k-1} x=0$. Hence $k=1$ and $p x=0$. Evidently $R$ is a direct sum of the $R_{p}, p \in \pi$.

LEMMA 13. If a regular ring $R$ does not contain an additively periodic element then $R$ is an algebra over the field of rationals.

Proof. Take any $x \in R$ and any natural $n$. There exists $y \in R$ such that $n x y n x=n x$. Since $R$ has no additively periodic element, $n x y x=x$. Therefore for any $x \in R$ and any rational $m / n$ there exists $z \in R$ (obviously, unique) such that $n z=m x$. We can define a multiplication by rationals on the elements of $R$ by putting $(m / n) x=z$. Thereby $R$ will be made an algebra over $Q$.

Proof of Theorem 1. If $S$ is not periodic then the result follows by Lemma 9. Let $S$ be periodic. First we will prove that $I[H]$ is regular.

Denote by $P$ the set of periodic elements of $I$. Then $P$ is an ideal of $R$, and so $P$ is a regular ring whose additive group is periodic. Lemma 12 shows that $P$ is of the form $\bigoplus_{p \in \pi} A_{p}$, where each $A_{p}$ is an algebra over a field of characteristic $p>0$. Further, each $A_{p}$ is an ideal of $P$ and is therefore regular; and we can restrict $\pi$ to be the set of all primes that divide the additive periods of elements of $P$. Given that, for all $p \in \pi, p$ is not the order of an element in $H$, Lemma 3 shows that $A_{p}[H]$ is regular. Hence $P[H]$ is regular. Further, $I[H] / P[H] \cong(I / P)[H]$ and $I / P$ has no element which is additively periodic. By Lemmas 13 and $3,(I / P)[H]$ is regular. Therefore $I[H]$ is regular, too.

Now we will prove that $\rho(R[H])=I[H]$. It suffices to show that $\rho(R[H] / I[H])=0$, that is, to show that $\rho((R / I)[H])=0$. Denote by $\pi$ the set of primes which are orders of some elements in $H$. Let $K$ be the class of rings which do not contain any element whose additive period is in $\pi$. Then $I$ is the largest regular ideal of $R$ belonging to $K$. Clearly $K$ is closed under extensions. In particular, if $J$ is an ideal of $R$ such that $J / I$ is regular and belongs to $K$ then $J \in K, J$ is regular, whence $J=I$. Therefore to simplify the notation we may assume that $I=0, R=R / I$.

Suppose that $\rho(R[H]) \neq 0$ and take any nonzero $x \in \rho(R[H])$, say $x=\sum_{i=1}^{n} x_{i} h_{i}$, where $x_{i} \in R, x_{i} \neq 0, h_{i} \in H$. Assume that $x$ is chosen so that $n$ is the least possible number here. Let $J$ denote the ideal generated in $R$ by $x_{n}$. Since $I=0$, it follows that $J$ contains an element whose additive period is divisible by a prime $p$ that is the order of an element in $H$. Hence $H$ has a subgroup with $p$ elements for the prime $p$ such that $J$ contains an element $y$ of additive period $p$, say $y=m x_{n}+a x_{n}+x_{n} b+\sum_{j=1}^{n} a_{j} x_{n} b_{j}$, where $m$ is an integer and $a, b, a_{j}, b_{j} \in R$. Set $z=m x+a x+x b+\sum_{j=1}^{k} a_{j} x b_{j}, R_{p}=\{r \in R \mid p r\}=0$. Note that $z \neq 0$, since $y \neq 0$. Further, $z \in \rho(R[H])$, because $x$ is taken in $\rho(R[H])$ and $z$ belongs to the ideal generated by $x$ in $R[H]$, moreover $z \in R^{1} x R^{1}$. Since $p y=0$, we see that $p z$ has a smaller number of summands than $z$, and so $p z=0, z \in R_{p}[H]$. Therefore $z \in \rho\left(R_{p}[H]\right)$. 
Denote by $G$ the subgroup generated in $H$ by $h_{1}, \ldots, h_{n}$. Since $S$ is periodic, then $G$ is a finite Abelian group. By Lemma 6, $z \in \rho\left(R_{p}[G]\right)$. However, Lemma 10 implies $\rho\left(R_{p}[H]\right)=0$. The contradiction shows that $\rho(R[H])=I[H]$.

Further, given that $S$ is Archimedean, for each $t \in S$, there is an $n$ such that $t^{n} \in e S=H$, where $e$ is the identity of $H$. Therefore the ideal generated in $R[S]$ by the set $R t$ is nilpotent modulo $R[H]$. Hence $R[S] / R[H]$ is a sum of nilpotent ideals, and so $\rho(R[S]) \subseteq \rho(R[H])$. The heredity of $\rho$ yields $\rho(R[S])=\rho(R[H])=I[H]$. The theorem is proved.

2. The general case. In this section, an arbitrary commutative semigroup $S$ will be considered. We shall need the following concept. A commutative semigroup $\Gamma$ is called a semilattice if it entirely consists of idempotents. We say that $S$ is a semilattice $\Gamma$ of its subsemigroups $S_{\alpha}, \alpha \in \Gamma$, if and only if $S=\bigcup_{\alpha \in \Gamma} S_{\alpha}, S_{\alpha} \cap S_{\beta}=\varnothing$ whenever $\alpha \neq \beta$ and $S_{\alpha} S_{\beta} \subseteq S_{\alpha \beta}$ for any $\alpha, \beta \in \Gamma$ (see [1]).

Now we fix some notation. Let $R$ be a ring, $S$ a commutative semigroup. It is known [1, Theorem 4.18] that $S$ is a semilattice $\Gamma$ of Archimedean subsemigroups $S_{\alpha}(\alpha \in \Gamma)$. Let $x \in R[S], x=\sum_{t \in S} x_{t} t$. For any $\alpha \in \Gamma$, set $x_{\alpha}=\sum_{t \in S_{\alpha}} x_{t} t$. The semilattice supp $(x)$ generated in $\Gamma$ by all $\alpha$ such that $x_{\alpha} \neq 0$ will be called the support of $x$. Consider the natural partial order $\leqslant$ on $\Gamma$ defined by the rule $\alpha \leqslant \beta \Leftrightarrow \alpha \beta=\alpha$. Let $\max (x)$ denote the set of maximal elements in $\operatorname{supp}(x)$. It is known that $\Gamma$ is locally finite, so $\operatorname{supp}(x)$ and $\max (x)$ are finite.

THEOREM 2. The radical $\rho(R[S])$ is the largest ideal among ideals $I$ in $R[S]$ such that $x_{\mu} \in \rho\left(R\left[S_{\mu}\right]\right)$ for any nonzero $x \in I, \mu \in \max (x)$.

This theorem follows from the more general result of [5]. For the sake of completeness we adduce a separate more simple proof.

Proof. Let $M$ denote the set of ideals $I$ in $R[S]$ such that $x_{\mu} \in \rho\left(R\left[S_{\mu}\right]\right)$ for any nonzero $x \in I, \mu \in \max (x)$. By [16, proof of Theorem 1], $\rho(R[S]) \in M$. Now take any $I$ from $M$. We will show that $I$ is regular. This will mean that $I \subseteq \rho(R[S])$.

Pick any $x \in I$ and set $n=|\operatorname{supp}(x)|$. We show by induction on $n$ that there is $y$ in $R$ such that $x y x=x$. The case where $n=0$ is trivial. Assume $n>0$. If $\mu \in \max (x)$ then $x_{\mu} \in \rho\left(R\left[S_{\mu}\right]\right)$ and therefore $x_{\mu} z x_{\mu}=x$ for some $z \in R\left[S_{\mu}\right]$. Putting $u=x-x z x$, we get $u \in I$ and $|\operatorname{supp}(u)|<n$. Hence $u v u=u$ for some $v$. Therefore

$$
x=u+x z x=u v u+x z x=x(v-v x z-z x v+z x v x z+z) x .
$$

Thus $\rho(R[S])$ is the largest ideal in $M$, as asserted.

THEOREM 3. There exists a commutative semigroup $S=\bigcup_{\alpha \in \Gamma} S_{\alpha}$ such that for any field $F$ each ring $F\left[S_{\alpha}\right]$ contains a nonzero regular ideal but $\rho(F[S])=0$.

Proof. Consider the set $\mathbb{Z}$ of all integers endowed with the multiplication $i j=$ $\min \{i, j\}$. It is easy to see that $\mathbb{Z}$ is a semilattice. Set $S_{i}=\left\{n_{i}, e_{i}\right\}, S=\bigcup_{i \in \mathbb{Z}} S_{i}$ and define a commutative multiplication on $S$ by putting (for each $i<j$ ) $e_{j} n_{i}=n_{j} n_{i}=n_{i}, e_{j} e_{i}=n_{j} e_{i}=e_{i}$, $e_{i}=e_{i}^{2}=e_{i} n_{i}=n_{i}^{2}$. Straightforward verification shows that $S$ is a semigroup and is a semilattice $\mathbb{Z}$ of the $S_{i}$. It is clear that $\rho\left(F\left[S_{i}\right]\right)=F e_{i}$ for each field $F$. We have to prove that $\rho(F[S])=0$. 
Suppose that there is nonzero $x \in \rho(R[S])$, say $x=\sum_{i \in \mathbb{Z}} x_{i}$, where $x_{i}=f_{i} e_{i}+g_{i} n_{i}$, $f_{i}, g_{i} \in F$. Let $m$ be the largest integer such that $x_{m} \neq 0$. Theorem 2 implies $x_{m} \in \rho\left(F\left[S_{m}\right]\right)$, whence $g_{m}=0$. Further, $x n_{m-1}=f_{m} n_{m-1}+f e_{m-1}+\sum_{i<m-1} x_{i}$, where $f=x_{m-1} n_{m-1} \in F e_{m-1}$. Since $f_{m} \neq 0$ and all the summands of $x n_{m-1}$, except $f_{m} n_{m-1}$, do not involve $n_{m-1}$, it is clear that $f_{m} n_{m-1}$ cannot be cancelled, so $x n_{m-1} \neq 0$. By Theorem 2,

$$
f_{m} n_{m-1}+f e_{m-1}=\left(x n_{m-1}\right)_{m-1} \in \rho\left(F\left[S_{m-1}\right]\right)=F e_{m-1},
$$

and therefore $n_{m-1}=e_{m-1}$. The contradiction completes our proof.

The results of [9] show that there is not any analogous example for the Jacobson radical. If we take a semigroup ring over an arbitrary ring of coefficients then the following question seems natural and rather difficult.

Question. Do there exist a ring $R$ and a commutative semigroup $S=\bigcup_{\alpha \in \Gamma} S_{\alpha}$ such that all the $R\left[S_{\alpha}\right]$ are not Jacobson semisimple but $R[S]$ is semisimple?

Note that, in [16] and [14], examples of a non-commutative $S=\bigcup_{\alpha \in \Gamma} S_{\alpha}$ were constructed such that $R[S]$ is Jacobson semisimple while there is only one semisimple ring among the $R\left[S_{\alpha}\right], \alpha \in \Gamma$. An analogous example of a non-commutative $S$ where all $R\left[S_{\alpha}\right]$ are not semisimple can be constructed with the use of the well-known Formanek's example [13, Theorem 7.4.8].

We will point out one more difference between the descriptions for the regular radical and the Jacobson one. In [5], the Jacobson radical $J(R[S])$ for a commutative $S$ was characterized with the use of so called simplest elements. These elements were earlier applied to the investigations of other semigroup rings in [15]. In particular it was proved that every simplest element lies in $J(R[S])$. Now we will show that a simplest element may not belong to the regular radical $\rho(R[S])$. So it is hardly possible to get a description of $\rho(R[S])$ in terms of simplest elements in the general case.

Let $\Omega$ be the set of $\alpha \in \Gamma$ such that $S_{\alpha}$ has an idempotent $e_{\alpha}$. If $\mu \in \Omega, x \in R\left[S_{\mu}\right]$ and $\Lambda$ is a finite (possibly empty) subset of $\mu \Omega$ then set $(\mu, x, \Lambda)=x \prod_{\lambda \in \Lambda}\left(e_{\mu}-e_{\lambda}\right)$. Here $(\mu, x, \Lambda)=x$ for $\Lambda=\varnothing$. If, moreover, $x \in \rho\left(R\left[S_{\mu}\right]\right)$ and $x t \in \rho\left(R\left[S_{\alpha}\right]\right)$ for each $\alpha \in \mu \Gamma \backslash$ $\Lambda \Gamma, t \in S_{\alpha}$ then $(\mu, x, \Lambda)$ is said to be a $\rho$-simplest element of $R[S]$. It follows from [15] that $J$-simplest elements belong to $J(R[S])$. However, the ring $F[S]$ constructed in the proof of Theorem 3 contains a $\rho$-simplest element $e_{2}-e_{1}$ which does not belong to $\rho(F[S])=0$.

Following [7], we say that $\rho$ is $S$-invariant if $\rho(R[S])=\rho(R)[S]$ for each $R$.

Corollary 1. Let $S$ be a commutative semigroup, $R$ a ring. The regular radical $\rho(R[S])$ is equal to $\rho(R)[S]$ if and only if $S$ is a union of finite groups whose orders are not divisible by the additive period of any element from $\rho(R)$.

Proof. Let $S=\bigcup_{\alpha \in \Gamma} S_{\alpha}, S_{\alpha}$ the Archimedean components of $S$. Set $F=R / \rho(R)$. By Theorem 1, $\rho\left(F\left[S_{\alpha}\right]\right)=0$. Theorem 2 implies $\rho(F[S])=0$; so $\rho(R[S]) \subseteq \rho(R)[S]$. Therefore $\rho(R[S])=\rho(R)[S]$ is equivalent to the fact that $\rho(R)[S]$ is regular. 
If $S$ is a union of finite groups whose orders are not divisible by the additive period of any element from $\rho(R)$ then the same can be said of every $S_{\alpha}$. Hence Theorem 1 implies $\rho\left(R\left[S_{\alpha}\right]\right)=\rho(R)\left[S_{\alpha}\right]$. Then for each nonzero $x \in \rho(R)[S]$ and each $m \in \max (x)$, it is clear that $x_{m} \in \rho(R)\left[S_{m}\right]=\rho\left(R\left[S_{m}\right]\right)$. By Theorem 2, $\rho(R)[S] \subseteq \rho(R([S])$, and therefore $\rho(R)[S]=\rho(R[S])$.

Suppose that $S$ is not a union of finite groups whose orders are not divisible by the additive period of any element from $\rho(R)$. Then, by Theorem $1, \rho(R)\left[S_{\alpha}\right]$ is not regular for some $\alpha \in \Gamma$. Take $x \in \rho(R)\left[S_{\alpha}\right] \rho\left(R\left[S_{\alpha}\right]\right)$. Then $\alpha \in \max (x), x \in \rho(R)[S]$, but $x_{\alpha} \notin$ $\rho\left(R\left[S_{\alpha}\right]\right)$. Theorem 2 implies that $\rho(R)[S]$ is not regular. This completes the proof.

Corollary 1 immediately gives us the following result.

Corollary 2. The regular radical is $S$-invariant if and only if $S$ is a semilattice.

Strangely enough the same class of semigroups answers the question when the Jacobson radical is $S$-invariant (see [6]). For a non-commutative $S$, the corresponding questions involve rather difficult problems in the case of characteristic $p>0$. It is still not known when the group ring of a locally finite group is Jacobson semisimple and when a semigroup ring is regular (see [13], [10]).

\section{REFERENCES}

1. A. H. Clifford and G. B. Preston, The algebraic theory of semigroups, Vol. 1, Mathematical Surveys No. 7 (American Mathematical Society, 1961).

2. K. R. Goodearl, von Neumann regular rings (Pitman, 1979).

3. E. Jespers and P. Wauters, A description of the Jacobson radical of semigroup rings of commutative semigroups, Group and semigroup rings, Proceedings of Conference, Johannesburg, 1985, Ed. G. Karpilovsky (North-Holland, 1966), 43-89.

4. A. V. Kelarev, On regular semigroup rings, Semigroup Forum 40 (1990), 113-114.

5. A. V. Kelarev, A description of the radicals of semigroup algebras of commutative semigroups, to appear.

6. A. V. Kelarev, On the Jacobson radical of semigroup rings of commutative semigroups, Math. Proc. Cambridge Philos. Soc. 108 (1990), 429-433.

7. J. Krempa, Radicals of semigroup rings, Fund. Math. 85 (1974), 57-71.

8. W. D. Munn, On the regularity of certain semigroup algebras, Semigroups, Proceedings of the Conference held at Monash University, 1979, Ed. T. E. Hall, P. R. Jones and G. B. Preston (Academic Press, 1980), 207-224.

9. W. D. Munn, On commutative semigroup algebras, Math. Proc. Cambridge Philos. Soc. 93 (1983), 237-246. $145-151$.

10. J. Okniński, On regular semigroup rings, Proc. Roy. Soc. Edinburgh Sect. A 99 (1984),

11. J. Okninski, Semigroup rings as excellent extensions and the regular radical, Simon Stevin 61 (1987), 301-311.

12. D. S. Passman, The algebraic structure of group rings (Wiley-Interscience, 1977).

13. D. S. Passman, Group rings, crossed products and Galois theory, CBMS Regional Conference Series in Mathematics, 64 (American Mathematical Society, 1986).

14. I. S. Ponizovskî, An example of a semiprimitive semigroup algebra, Semigroup Forum 26 (1983), 225-228. 
15. I. S. Ponizovskiî, On semigroups rings, Semigroup Forum 28 (1984), 143-154.

16. M. L. Teply, E. G. Turman and A. Quesada, On semisimple semigroup rings, Proc. Amer. Math. Soc. 79 (1980), 157-163.

17. J. Weissglass, Regularity of semigroup rings, Proc. Amer. Math. Soc. 25 (1970), 499-503.

Department of Mathematics and Mechanics

URAL STATE UNIVERSITY

LENINA 51

EKATHERIMBURG 620083

RusSIA 